\title{
Aspergillus fumigatus during stable state and exacerbations of COPD
}

\author{
Mona Bafadhel ${ }^{1}$, Susan Mckenna², Joshua Agbetile², Abbie Fairs², \\ Dhananjay Desai², Vijay Mistry², Joseph P. Morley², Mitesh Pancholi², \\ Ian D. Pavord', Andrew J. Wardlaw², Catherine H. Pashley ${ }^{2,3}$ and \\ Christopher E. Brightling 2,3
}

\author{
Affiliations: \\ 'Dept of Respiratory Medicine, Nuffield Department of Medicine, University of Oxford, Oxford, and \\ ${ }^{2}$ Dept of Infection, Immunity and Inflammation, Institute for Lung Health, NIHR Respiratory Biomedical \\ Research Unit, University of Leicester, Leicester, UK. \\ ${ }^{3}$ Both authors contributed equally.
}

Correspondence: M. Bafadhel, Dept of Respiratory Medicine, Nuffield Dept of Medicine, University of Oxford, Old Road Campus, Oxford, OX3 7FZ, UK. E-mail: mona.bafadhellandm.ox.ac.uk

ABSTRACT Bacteria are often isolated in stable chronic obstructive pulmonary disease (COPD). Whether fungi are also commonly present and associated with clinical and pathological features of disease is uncertain. We investigated the frequency of filamentous fungal culture and IgE sensitisation to Aspergillus fumigatus and the relationship to clinical outcomes in COPD subjects.

COPD subjects were recruited to enter a 1-year observational study. Assessments of lung function, allergen testing and sputum analysis for inflammation, bacteria and fungus were undertaken in COPD subjects and healthy smoking and nonsmoking controls.

Filamentous fungi were cultured at baseline in 49\% (63 out of 128) of COPD subjects, of which $75 \%$ ( 47 out of 63) were A. fumigatus. Fungus was cultured in three out of 22 controls (two were A. fumigatus). The total sputum cell count and inhaled corticosteroid dosage were significantly increased in COPD patients with a positive filamentous fungal culture at baseline $(p<0.05)$. Sensitisation to A. fumigatus was present in $13 \%$ of COPD subjects and was associated with worse lung function (forced expiratory volume in $1 \mathrm{~s} 39 \%$ predicted versus $51 \%$ predicted; $\mathrm{p}=0.01$ ), but not related to filamentous fungal culture.

A. fumigatus sensitisation is related to poor lung function. Positive filamentous fungal culture is a common feature of COPD. The clinical significance of this remains uncertain.

@ERSpublications

A. fumigatus sensitisation links to poor COPD lung function; clinical significance of positive fungal culture is unclear http://ow.ly/qfr5q

\footnotetext{
This article has supplementary material available from www.erj.ersjournals.com

Earn CME accreditation by answering questions about this article. You will find these at the back of the printed copy of this issue or online at www.erj.ersjournals.com/misc/cmeinfo.xhtml

Received: Oct 112012 | Accepted after revision: April 102013 | First published online: April 182013

Support statement: The study was funded by the Medical Research Council (UK), a senior Wellcome Fellowship (C.E. Brightling) and the Midlands Asthma Allergy Research Association. The research was performed in laboratories part funded by the European Regional Development Fund (ERDF 05567). This article presents independent research funded by the National Institute for Health Research (NIHR). The views expressed are those of the authors and not necessarily those of the NHS, the NHR or the Department of Health.
}

Conflict of interest: Disclosures can be found alongside the online version of this article at www.erj.ersjournals.com

Copyright @ERS 2014 


\section{Introduction}

Chronic obstructive pulmonary disease (COPD) is associated with significant morbidity and mortality [1]. It is characterised by irreversible airflow obstruction [2] with underlying emphysema and small airway obliteration, which co-exist commonly $[3,4]$. Airways of patients with COPD are often "colonised" with potential pathogenic microorganisms [5] which give rise to increased airway inflammation [6]. Bacteria and viruses have been implicated as the major cause of COPD exacerbations, whereas the potential role of fungal colonisation and infection in the pathogenesis of COPD is poorly understood. The commonest fungal genus to cause pulmonary-associated fungal infections is Aspergillus [7], with a wide spectrum of syndromes, including saprophytic invasion, allergic disease and invasive aspergillosis [8], often due to Aspergillus fumigatus [7]. Additionally, allergic bronchopulmonary aspergillosis (ABPA) [9-12], found commonly in asthma and cystic fibrosis [13], is increasingly recognised in COPD [14]. Furthermore, sensitisation to A. fumigatus has been found to be associated with poor lung function in severe asthmatics [15]. Impairment in host defence systems in immunocompetent and immunocompromised patients, including those with COPD, is thus likely to promote susceptibility to fungal infections. How this impacts on important clinical outcomes in COPD such as disease severity, inflammation and exacerbations is currently unknown.

We hypothesised that the presence of filamentous fungi in the airways of patients with COPD and sensitisation to A. fumigatus is associated with disease severity, airway inflammation and exacerbations. To study this, we carried out a longitudinal study to investigate fungal culture in patients with COPD during stable state and exacerbations.

\section{Methods}

Subjects and measurements

Subjects with COPD diagnosed according to the Global Initiative for Chronic Obstructive Lung Disease (GOLD) guidelines [2] were recruited as part of a larger biomarker-directed randomised controlled study [16]. Subjects with a diagnosis of asthma, active pulmonary tuberculosis or any other clinically relevant lung disease were excluded. All COPD subjects underwent lung function testing including reversibility testing [17]. Sputum was collected for analysis of routine microbiology for potential pathogenic microorganisms (defined as Haemophilus influenzae, Moraxella catarrhalis, Streptococcus pneumoniae, Staphylococcus aureus or Pseudomonas aeruginosa) [18] and mycology [15, 19]. In brief, $\sim 150 \mathrm{mg}$ of undiluted sputum was inoculated onto potato dextrose agar plates containing chloramphenicol $\left(16 \mu \mathrm{g} \cdot \mathrm{mL}^{-1}\right)$, gentamicin $\left(4 \mu \mathrm{g} \cdot \mathrm{mL}^{-1}\right)$ and fluconazole $\left(5 \mu \mathrm{g} \cdot \mathrm{mL}^{-1}\right)$. Plates were then incubated at $37^{\circ} \mathrm{C}$ and inspected daily for up to 7 days. A. fumigatus colonies were identified by colony formation and microscopy. PCR sequencing of the large subunit or the internal transcribed spacer region of the nuclear ribosomal operon was used for the detection of other filamentous fungi, as previously described [19].

Sputum was also processed to produce cytospins for assessment of sputum total and differential cell counts $[20,21]$. Venous blood was collected for assessment of peripheral blood differential cell counts and serum C reactive protein. Health status and symptom scores were measured using the St George's Respiratory Questionnaire (University of London, London, UK) [22], the Chronic Respiratory Disease InterviewerAdministered Questionnaire (CRQ) (McMaster University, Hamilton, Canada) [23] and the visual analogue scale (VAS) for the domains of cough, breathlessness, sputum production and sputum purulence [24]. Subjects were seen during stable-state and exacerbation visits; sample collection was performed prior to

TABLE 1 Clinical characteristics of chronic obstructive pulmonary disease (COPD) subjects and controls

COPD subjects

Subjects n
Male
Age years
Smoking pack-years
Atopy \%
FEV1\% predicted
Filamentous fungal culture positive
Aspergillus fumigatus culture

Data presented as $\mathrm{n}(\%)$, mean (range) or mean \pm SEM, unless otherwise stated. FEV1: forced expiratory volume in $1 \mathrm{~s}$. 
TABLE 2 Clinical characteristics of chronic obstructive pulmonary disease subjects according to filamentous fungal status

\section{No fungus culture \\ A. fumigatus culture \\ Other filamentous fungus} culture

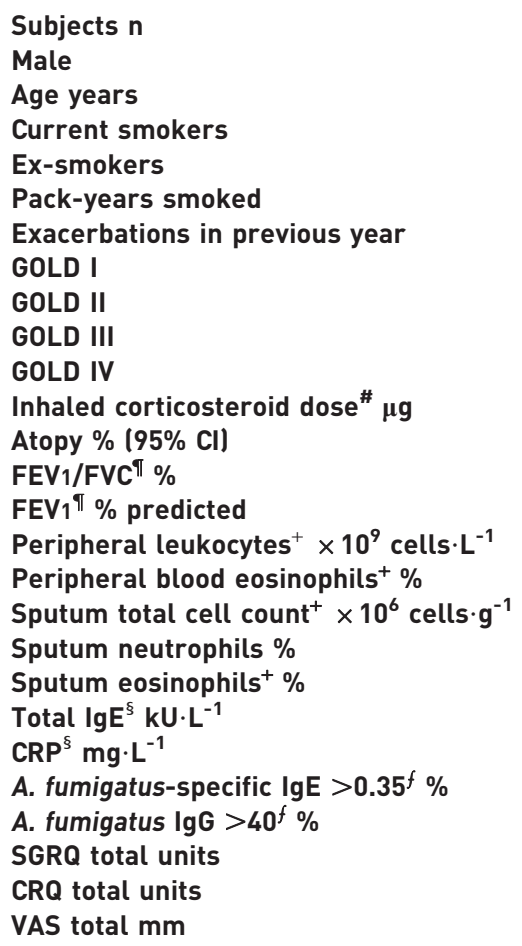

47
$35(75)$
$72(53-86)$
$11(23)$
$34(72)$
$49(10-130)$
$3(1-8)$
$2(4)$
$16(34)$
$16(34)$
$13(28)$
$1628 \pm 84$
$55(38-70)$
$49 \pm 2$
$48 \pm 2$
$7.8(7.3-8.4)$
$2.5(2.1-3.0)$
$4.1(2.9-5.9)$
$78 \pm 3$
$0.8(0.6-1.2)$
$57.7 \pm 165.5$
$3 \pm 8$
15
28
$53 \pm 2$
$4.2 \pm 0.2$
$162 \pm 13$

$\begin{array}{cc}16 & \\ 13(81) & 0.24 \\ 68(51-82) & 0.10 \\ 6(37) & \mathbf{0 . 0 3} \\ 10(63) & 0.07 \\ 57(12-138) & 0.56 \\ 3(1-10) & 0.30 \\ 1(6) & 0.95 \\ 3(18) & 0.22 \\ 6(38) & 0.76 \\ 6(38) & 0.58 \\ 1754 \pm 130 & \mathbf{0 . 0 5} \\ 14(1-53) & 0.07 \\ 46 \pm 4 & 0.55 \\ 44 \pm 5 & 0.38 \\ 7.9(6.8-9.2) & 0.12 \\ 2.5(1.7-3.8) & 0.56 \\ 4.1(2.8-5.9) & \mathbf{0 . 0 3} \\ 78 \pm 6 & \mathbf{0 . 0 3} \\ 1.7(0.7-4.2) & 0.19 \\ 46.9 \pm 151.4 & 0.64 \\ 5 \pm 9 & 0.65 \\ 0 & 0.26 \\ 15 & 0.26 \\ 47 \pm 4 & 0.14 \\ 4.4 \pm 0.2 & 0.08 \\ 157 \pm 11 & 0.90\end{array}$

Data are presented as $\mathrm{n}(\%)$, mean (range) or mean \pm SEM, unless otherwise stated. Bold type represents statistical significance at $\mathrm{p}<0.05$. SGRQ scores ranging from 0 to 100 with a higher score indicating worse health status (total score made up of impact, symptoms and activity domains); $\mathrm{CRQ}$ scores range from 1 to 7 , with a higher score representing better health quality (total score made up of emotion, dyspnoea, fatigue and mastery domains); VAS performed on a 100-mm line from "no symptoms" to "worst symptoms", higher scores represent worse symptoms (total score calculated as addition of cough, dyspnoea, sputum volume production and purulence domains). A. fumigatus: Aspergillus fumigatus; GOLD: Global Initiative for Chronic Obstructive Lung Disease stage; FEV1: forced expiratory volume in 1 s; FVC: forced vital capacity; CRP: C reactive protein; SGRQ: St George's Respiratory Questionnaire; CRQ: Chronic Respiratory Health Questionnaire; VAS: visual analogue scale. ${ }^{\#}$ : beclomethasone dipropionate equivalent; ${ }^{\uparrow}$ : post-bronchodilator; ${ }^{+}$: geometric mean $\left(95 \%\right.$ confidence interval); ${ }^{\S}$ : median (interquartile range); ${ }^{f}$ : proportion $\lg E>0.35$ or $\lg G>40$.

randomisation and institution of any therapy. Nonsmoking and nonobstructed smoking controls were invited to attend a single study visit, with lung function testing, sputum induction and demographic data collection. Skin-prick testing was used to assess atopy to Dermatophagoides pteronyssinus, dog, cat, grass pollen and to the fungi Alternaria alternata, A. fumigatus, Botrytis cinerea, Cladosporium herbarum and Penicillium chrysogenum (Alk-Abello, Hørsholm, Denmark). Total IgE levels, allergen-specific IgE antibody levels to cat, dog, timothy grass, D. pteronyssinus and A. fumigatus, and A. fumigatus-IgG levels were measured using the ImmunoCap 250 system (Phadia Ltd, Milton Keynes, UK).

The study was approved by the Leicestershire, Northamptonshire and Rutland ethics committee and all subjects gave informed written consent.

\section{Statistical analysis}

Statistical analysis was performed using PRISM version 4 (GraphPad, San Diego, CA, USA) and SPSS version 16 (SPSS Inc., Chicago, IL, USA). Parametric and nonparametric data are presented as mean \pm SEM and median (interquartile range) unless otherwise stated. Log-transformed data are presented as geometric mean $(95 \% \mathrm{CI})$. For comparison of unpaired or paired parametric or nonparametric groups, t-tests, MannWhitney U-test and Wilcoxon matched-pairs tests were used. For comparison of three groups or more for parametric and nonparametric variables the ANOVA or Kruskal-Wallis test was used, and the Chi-squared test was used for proportions. Repeatability of A. fumigatus culture was assessed in patients with two stable 

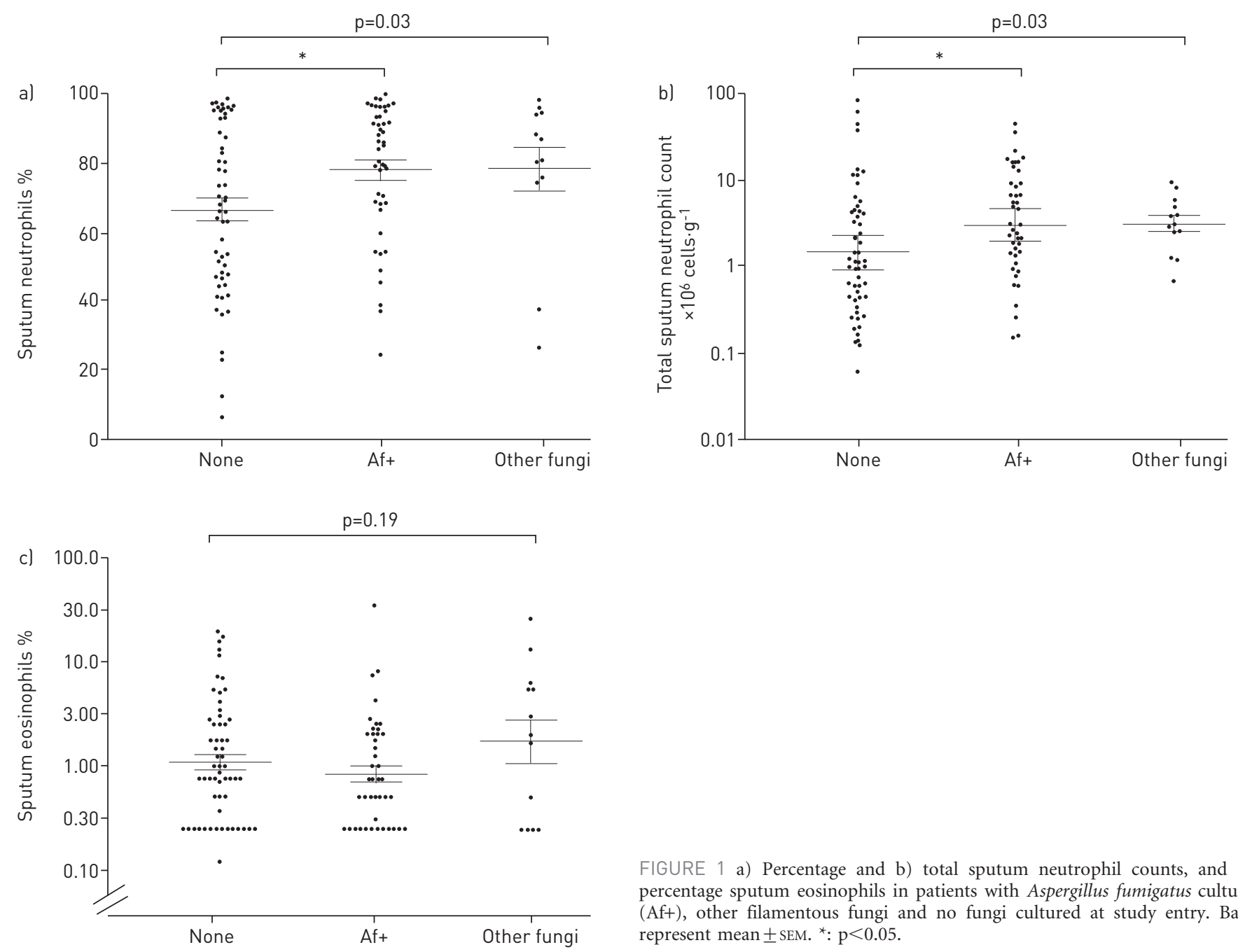

FIGURE 1 a) Percentage and b) total sputum neutrophil counts, and c) percentage sputum eosinophils in patients with Aspergillus fumigatus culture $(\mathrm{Af}+)$, other filamentous fungi and no fungi cultured at study entry. Bars represent mean \pm SEM. ${ }^{*}: \mathrm{p}<0.05$.

visits 3 months apart, using the Cohen $\kappa$ statistic. Logistic regression analysis was used to assess the relationship of the variables of forced expiratory volume in $1 \mathrm{~s}$ (FEV1) \% predicted, sputum eosinophil count (log-transformed), sputum neutrophil count (log-transformed) and exacerbation frequency with the presence of 1) A. fumigatus-only culture and 2) sensitisation to A. fumigatus using the block entry method. Standard multiple regression analysis was used to assess the relationship of airway inflammation, exacerbation frequency, fungal culture and fungal sensitisation with lung function. Logistic regression goodness-to-fit was performed using the Hosmer-Lemeshow Chi-squared and R-squared (true and pseudo$\mathrm{R}^{2}$ for multiple and logistic regression, respectively) tests were used to estimate the variance explained by the model. A p-value of $<0.05$ was taken as the threshold of significance.

TABLE 3 Predictors of Aspergillus fumigatus sputum culture in chronic obstructive pulmonary disesase subjects at stable state

OR $(95 \% \mathrm{Cl})$

p-value

\begin{tabular}{lll}
\hline Exacerbation frequency & $0.84(0.69-1.03)$ & 0.09 \\
FEV1\% predicted & $0.97(0.96-1.01)$ & 0.32 \\
Sputum eosinophils \% & $0.53(0.24-1.20)$ & 0.13 \\
Total sputum neutrophil count & $1.97(1.05-3.69)$ & $\mathbf{0 . 0 3}$ \\
\hline
\end{tabular}

Bold type represents statistical significance at $p<0.05$. FEV1: forced expiratory volume in $1 \mathrm{~s}$. 


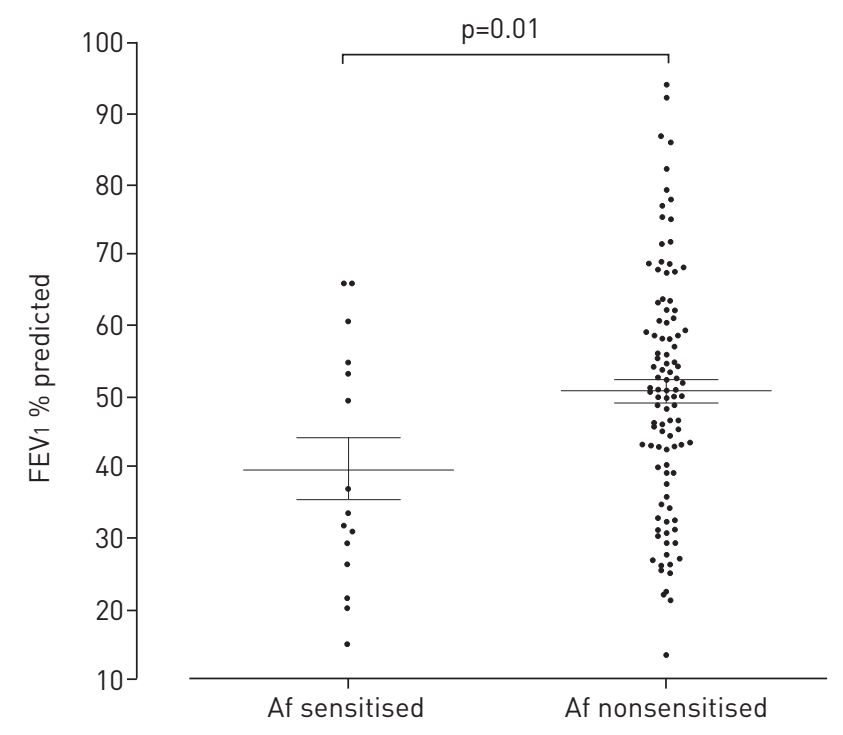

FIGURE 2 Forced expiratory volume in $1 \mathrm{~s}$ (FEV1) \% predicted categorised according to Aspergillus fumigatus (Af) sensitisation. Bars represent mean \pm SEM.

\section{Results}

Filamentous fungal culture and baseline demographic data were obtained in 128 patients with COPD (89 males and 39 females). The mean FEV 1 in the COPD subjects was $48 \pm 3 \%$ pred. Control data were available in 22 (eight males and 14 females) subjects with a mean (range) age of 58 (41-79) years; mean (range) smoking history of $10(0-30)$ pack-years and a mean FEV1 of $116 \pm 3 \%$ pred. The clinical characteristics of the COPD patients and controls are presented in table 1. A filamentous fungus was isolated in $49 \%$ (63 out of 128) of COPD patients and was predominantly Aspergillus or Penicillium species (online supplementary table E1). A. fumigatus was cultured in 37\% (47 out of 128) of patients, while identification of any Aspergillus species was present in $42 \%$ (55 out of 128) of patients. Pathogenic bacteria ( $H$. influenzae, M. catarhalis, S. pneumoniae, S. aureus or P. aeruginosa) and A. fumigatus co-culture was found in $14 \%$ (18 out of 128) of patients at baseline stable state. The proportion of control subjects that had positive filamentous fungal cultures was significantly lower compared to COPD subjects (14\% versus 49\%; mean difference $36 \%$, 95\% CI 14-48\%; $\mathrm{p}=0.002$ ).

\section{A. fumigatus culture and clinical outcomes}

Patients with A. fumigatus were on a higher inhaled corticosteroid dose compared to those who were culture-negative (1628 versus $1389 \mu \mathrm{g}$ beclomethasone dipropionate equivalent; mean difference 239, 95\% CI $0-477 ; \mathrm{p}=0.050)$. There were no differences in health status, exacerbation frequency or FEV1 \% pred in COPD subjects who were A. fumigatus culture-positive compared to those who were A. fumigatus culturenegative (table 2). There was a significant difference in total and percentage sputum neutrophil count, but not in sputum eosinophils in patients with A. fumigatus compared to patients with no fungi or other filamentous fungi (fig. 1). The total sputum neutrophil count was the most independent predictor of A. fumigatus culture, with a sensitivity and specificity of $54 \%$ and $76 \%$, respectively (table 3 ).

\section{Atopy and A. fumigatus}

Atopy, defined as a positive skin prick testing and/or elevated allergen-specific antibodies, was found in $34 \%$ of COPD patients. Concordance of serum-specific IgE antibodies and skin prick testing to the common allergens, showed a good strength of agreement $(\kappa=0.63,95 \%$ CI $0.49-0.76)$. Sensitisation to A. fumigatus (positive skin prick test and/or elevated A. fumigatus IgE antibodies) was present in 13\% of patients. Sensitisation to A. fumigatus, irrespective of corresponding filamentous fungal culture, was associated with lower lung function (FEV1 39\% pred versus 51\% pred; mean difference 11\%, 95\% CI 3-20\%; p=0.01) (fig. 2). There were no differences in parameters of airway inflammation, health status or exacerbation frequency in those that were or were not sensitised to A. fumigatus. FEV1 \% pred was the best predictor of A. fumigatus sensitisation (table 4).

\section{A. fumigatus colonisation}

The repeatability of $A$. fumigatus culture was examined in 70 subjects at two stable visits 3 months apart. The $\kappa$ agreement statistic $(95 \% \mathrm{CI})$ was $-0.04(-0.29-0.21)$. This was determined to be below that observed by chance alone. Of these patients, $13 \%$ and $39 \%$ were either A. fumigatus-positive or culture-negative, 
TABLE 4 Predictors of Aspergillus fumigatus sensitisation in chronic obstructive pulmonary disease subjects at stable state

\begin{tabular}{lll} 
& OR $(95 \% \mathrm{CI})$ & p-value \\
\hline Exacerbation frequency & $1.17(0.89-1.55)$ & 0.27 \\
FEV1\% predicted & $0.95(0.91-0.99)$ & $\mathbf{0 . 0 2}$ \\
Sputum eosinophils \% & $1.04(0.34-3.23)$ & 0.94 \\
Total sputum neutrophil count & $2.12(0.78-5.81)$ & 0.14 \\
Aspergillus fumigatus culture & $1.23(0.34-4.49)$ & 0.76 \\
\hline
\end{tabular}

Bold type represents statistical significance at $\mathrm{p}<0.05$. FEV1: forced expiratory volume in $1 \mathrm{~s}$.

respectively, at both. Neutrophil or eosinophil systemic and airway inflammatory measures were not different between patients who cultured A. fumigatus, other filamentous fungi and no fungi, neither in the repeated visit, nor in patients who either persistently grew filamentous fungi or never grew fungi on two visits.

\section{A. fumigatus during exacerbations}

110 exacerbations with filamentous fungal culture from 80 patients were captured. A positive filamentous fungal culture was found in $38 \%$ of all exacerbation events; while $28 \%$ of all exacerbation events were culture-positive to A. fumigatus. There was no difference in airway inflammation, change in CRQ, FEV1 or VAS scores between those who cultured A. fumigatus at exacerbation compared to those who did not. Corresponding filamentous fungal and bacterial culture data during exacerbations were available in 79 exacerbation events. Co-culture of A. fumigatus and pathogenic bacteria was observed in 14\% (11 out of 79) of exacerbation events. There were 44 and 35 bacteria- and nonbacteria-associated exacerbations of COPD. The acquisition of $A$. fumigatus was not different between bacteria-associated and nonbacterial-associated exacerbations $(20 \%$ versus $55 \%, \mathrm{p}=0.07)$. There was no change from baseline in lung function, health status and symptom scores in bacteria- or nonbacteria-associated exacerbations and the culture of A. fumigatus at the baseline and exacerbation visit (online supplementary tables E2 and E3).

\section{Discussion}

Here we report that in subjects with COPD, sensitisation to A. fumigatus was associated with poor lung function. Positive culture of filamentous fungi, especially that of A. fumigatus, was commonly found in the sputum of patients with COPD and was increased compared to controls. However, repeatability of A. fumigatus culture in stable state was poor and the prevalence did not change at exacerbations. The clinical significance of a positive filamentous fungal culture in COPD therefore remains uncertain.

In our study, hypersensitivity to A. fumigatus was detected in $13 \%$ of the COPD subjects and was associated with reduced lung function independent of $A$. fumigatus sputum culture. This is consistent with previously observed findings in patients with severe asthma using the same fungal culture technique [15]. Whether sensitisation to A. fumigatus contributes to the cause of airflow obstruction or is a consequence of a damaged and remodelled airway and thus more likely in subjects with severe COPD is uncertain. In severe asthma the relationship between sensitisation and filamentous fungal culture suggests that persistent colonisation may promote sensitisation. Although we were unable to replicate the finding of colonisation in our study, it remains plausible that sensitisation reflects increased filamentous fungi exposure over time.

Sensitisation to A. fumigatus is a feature of ABPA. Although we did not undertake computed tomography in this study, none of our subjects fulfilled the criteria of GREENBERGER and co-workers [11, 12] for ABPA. The incomplete fulfilment of these ABPA criteria has also been shown in previous COPD studies [14]. In asthma associated with ABPA and in severe asthma with fungal sensitisation, antifungal therapy has demonstrated clinical benefit, although the magnitude of this effect is small [25-27]. Whether antifungal therapy may be effective in reducing exacerbations and disease progression in COPD patients with filamentous fungal sensitisation remains to be tested.

Colonisation of the airways, defined as "the presence of potentially pathogenic organisms without an associated inflammatory response" is common in patients with COPD, occurring in up to 30\% [5]. Using a specialist fungal culture technique, we have shown that the presence of filamentous mould (mainly A. fumigatus) was found in almost half of subjects with COPD, and that neutrophilic airway inflammation was the best predictor of A. fumigatus sputum culture. The presence of A. fumigatus or any filamentous fungal culture was also related to higher doses of inhaled corticosteroids. Immunosuppression from 
corticosteroid treatment has been associated with invasive aspergillosis and studies have determined that corticosteroid therapy is an independent predictor of invasive aspergillosis in COPD patients in intensive care $[28,29]$. Current COPD guidelines advocate the use of inhaled corticosteroids in patients with FEV1 $<50 \%$ and recurrent exacerbations to reduce the risk of future exacerbations [2]. At present it remains unclear whether there is an inhaled corticosteroid dose-response predisposition to $A$. fumigatus colonisation in a susceptible cohort or an associated increase in risk of invasive aspergillosis; this requires further study.

Colonisation of Aspergillus in immunocompetent individuals does not fulfil the criteria for definite or probable invasive aspergillosis [30, 31]. However, this definition has focused on filamentous fungal detection from respiratory samples collected on a single episode. There is a paucity of data in the literature examining repeated A. fumigatus culture in COPD subjects at stable state, and even less investigating A. fumigatus during exacerbations. In this study we could not determine repeatability of A. fumigatus culture in subsequent stable visits. However, our study highlights the importance of examining relationships of pathogen and airway inflammation expression in longitudinal studies, and we have aimed to explore the classification of filamentous fungal colonisation in the COPD airway and related this to important clinical outcomes. Although we have been unable to show that there is persistence of A. fumigatus culture in a cohort of patients with repeated visits, emerging knowledge regarding the complex microbiota of the airways in COPD [32] would suggest that the role of filamentous fungi, and in particular A. fumigatus, needs to be further explored in the pathogenesis of COPD. Our study was strengthened by examining filamentous fungal culture in the airways of non-COPD controls, and showed that despite similar atopy rates there was significantly reduced detection of fungal and A. fumigatus culture in control subjects. Although the control group was not matched for age or smoking, the detection of filamentous fungus in the sputum was significantly lower and independent of atopy status. A final concern is whether we have inadvertently included patients with asthma and classed these patients as COPD. However, to reduce this bias a strict exclusion criteria for screening was a current or previous history of asthma, whether selfreported or physician-diagnosed, and used current spirometric diagnostic criteria for COPD as per GOLD guidelines [2]. Therefore, whether our finding of A. fumigatus sensitisation and worsened lung function is related to disease or prolonged exposure needs to be further evaluated in larger studies.

To our knowledge, this is the first study to examine filamentous fungal and A. fumigatus culture rates during exacerbations of COPD. In contrast to our hypothesis, we detected that filamentous fungal culture rates and in particular A. fumigatus was not increased during exacerbations of COPD. Studies have shown that bacterial and fungal interactions include promotion of survival and virulence of fungus, or inhibition of filament formation [33]. Furthermore, the development of bacterial and fungal biofilms may provide a host defence environment which has resistance to antibiotic properties [33]. In this study we found that A. fumigatus culture was not affected by the presence or absence of concomitant bacterial culture; whether interactions between filamentous fungal and bacterial infection during exacerbations are important remains to be fully determined.

In conclusion, we have shown that A. fumigatus in commonly found in the sputum of patients with COPD and that this is irrespective of disease severity. We have also shown that IgE sensitisation to A. fumigatus in COPD subjects is associated with lower lung function and that the detection of A. fumigatus by sputum culture is increased compared to controls but unrelated to exacerbations. However, the clinical significance of fungi in COPD and the response to antifungal therapy remains to be determined and further studies are required.

\section{Acknowledgements}

The authors would like to thank all the research volunteers who participated in the study and the following people for their assistance during the study: Michelle Bourne, Beverly Hargadon, Maria Shelley, Amisha Singapuri and Sarah Terry (Dept of Infection, Immunity and Inflammation, Institute for Lung Health, NIHR Respiratory Biomedical Research Unit, University of Leicester, Leicester, UK) and Hemu Patel (Dept of Clinical Microbiology, Leicester Royal Infirmary, University of Leicester Hospitals NHS Trust, Leicester, UK).

\section{References}

1 Lopez AD, Shibuya K, Rao C, et al. Chronic obstructive pulmonary disease: current burden and future projections. Eur Respir J 2006; 27: 397-412.

2 Rabe KF, Hurd S, Anzueto A, et al. Global strategy for the diagnosis, management, and prevention of chronic obstructive pulmonary disease: GOLD executive summary. Am J Respir Crit Care Med 2007; 176: 532-555.

Hogg JC, Chu F, Utokaparch S, et al. The nature of small-airway obstruction in chronic obstructive pulmonary disease. N Engl J Med 2004; 350: 2645-2653.

Jeffery PK. Structural and inflammatory changes in COPD: a comparison with asthma. Thorax 1998; 53: 129-136. Sethi S, Murphy TF. Infection in the pathogenesis and course of chronic obstructive pulmonary disease. $N$ Engl $J$ Med 2008; 359: 2355-2365. 
e8578.

33 Wargo MJ, Hogan DA. Fungal-bacterial interactions: a mixed bag of mingling microbes. Curr Opin Microbiol 2006; 9: $359-364$. Bulpa P, Dive A, Sibille Y. Invasive pulmonary aspergillosis in patients with chronic obstructive pulmonary disease. Eur Respir J 2007; 30: 782-800.

Al-Alawi A, Ryan CF, Flint JD, et al. Aspergillus-related lung disease. Can Respir J 2005; 12: 377-387.

Wardlaw A, Geddes DM. Allergic bronchopulmonary aspergillosis: a review. J R Soc Med 1992; 85: 747-751.

Tillie-Leblond I, Tonnel AB. Allergic bronchopulmonary aspergillosis. Allergy 2005; 60: 1004-1013.

Greenberger PA. Allergic bronchopulmonary aspergillosis. J Allergy Clin Immunol 1984; 74: 645-653.

Greenberger PA, Miller TP, Roberts M, et al. Allergic bronchopulmonary aspergillosis in patients with and without evidence of bronchiectasis. Ann Allergy 1993; 70: 333-338.

Denning DW. Chronic forms of pulmonary aspergillosis. Clin Microbiol Infect 2001; 7: Suppl. 2, $25-31$.

Agarwal R, Hazarika B, Gupta D, et al. Aspergillus hypersensitivity in patients with chronic obstructive pulmonary disease: COPD as a risk factor for ABPA? Med Mycol 2010; 48: 988-994.

Fairs A, Agbetile J, Hargadon B, et al. IgE sensitization to Aspergillus fumigatus is associated with reduced lung function in asthma. Am J Respir Crit Care Med 2010; 182: 1362-1368.

Bafadhel M, McKenna S, Terry S, et al. Blood eosinophils to direct corticosteroid treatment of exacerbations of chronic obstructive pulmonary disease: a randomized placebo-controlled trial. Am J Respir Crit Care Med 2012; 186: $48-55$.

Brusasco V, Crapo R, Viegi G. Coming together: the ATS/ERS consensus on clinical pulmonary function testing. Eur Respir J 2005; 26: 1-2. Standard Method BSOP 57 Issue 2.3.

Pashley CH, Fairs A, Morley JP, et al. Routine processing procedures for isolating filamentous fungi from respiratory sputum samples may underestimate fungal prevalence. Med Mycol 2012; 50: 433-438.

Pizzichini E, Pizzichini MM, Efthimiadis A, et al. Indices of airway inflammation in induced sputum: reproducibility and validity of cell and fluid-phase measurements. Am J Respir Crit Care Med 1996; 154 : 308-317. Pizzichini MM, Popov TA, Efthimiadis A, et al. Spontaneous and induced sputum to measure indices of airway inflammation in asthma. Am J Respir Crit Care Med 1996; 154: 866-869.

Jones PW, Quirk FH, Baveystock CM, et al. A self-complete measure of health status for chronic airflow limitation. The St. George's Respiratory Questionnaire. Am Rev Respir Dis 1992; 145: 1321-1327.

Guyatt G. Measuring health status in chronic airflow limitation. Eur Respir J 1988; 1: 560-564.

Brightling CE, Monterio W, Green RH, et al. Induced sputum and other outcome measures in chronic obstructive pulmonary disease: safety and repeatability. Respir Med 2001; 95: 999-1002.

Wark PA, Gibson PG, Wilson AJ. Azoles for allergic bronchopulmonary aspergillosis associated with asthma. Cochrane Database Syst Rev 2004; 3: CD001108.

Denning DW, O'Driscoll BR, Powell G, et al. Randomized controlled trial of oral antifungal treatment for severe asthma with fungal sensitization: The Fungal Asthma Sensitization Trial (FAST) study. Am J Respir Crit Care Med 2009; 179: 11-18.

Stevens DA, Schwartz HJ, Lee JY, et al. A randomized trial of itraconazole in allergic bronchopulmonary aspergillosis. N Engl J Med 2000; 342: 756-762.

Ader F, Nseir S, Le Berre R, et al. Invasive pulmonary aspergillosis in chronic obstructive pulmonary disease: an emerging fungal pathogen. Clin Microbiol Infect 2005; 11: 427-429.

McEvoy CE, Niewoehner DE. Adverse effects of corticosteroid therapy for COPD. A critical review. Chest 1997; 111: $732-743$.

Vandewoude KH, Blot SI, Depuydt P, et al. Clinical relevance of Aspergillus isolation from respiratory tract samples in critically ill patients. Crit Care 2006; 10: R31.

Lucena P, Barberán J, Eroles G, et al. Significance of lower respiratory tract cultures yielding Aspergillus spp. growth in a hospital without transplant patients. Rev Esp Quimioter 2010; 23: 190-195.

Hilty M, Burke C, Pedro H, et al. Disordered microbial communities in asthmatic airways. PLoS One 2010; 5:

O'Donnell R, Breen D, Wilson S, et al. Inflammatory cells in the airways in COPD. Thorax 2006; 61: 448-454. 